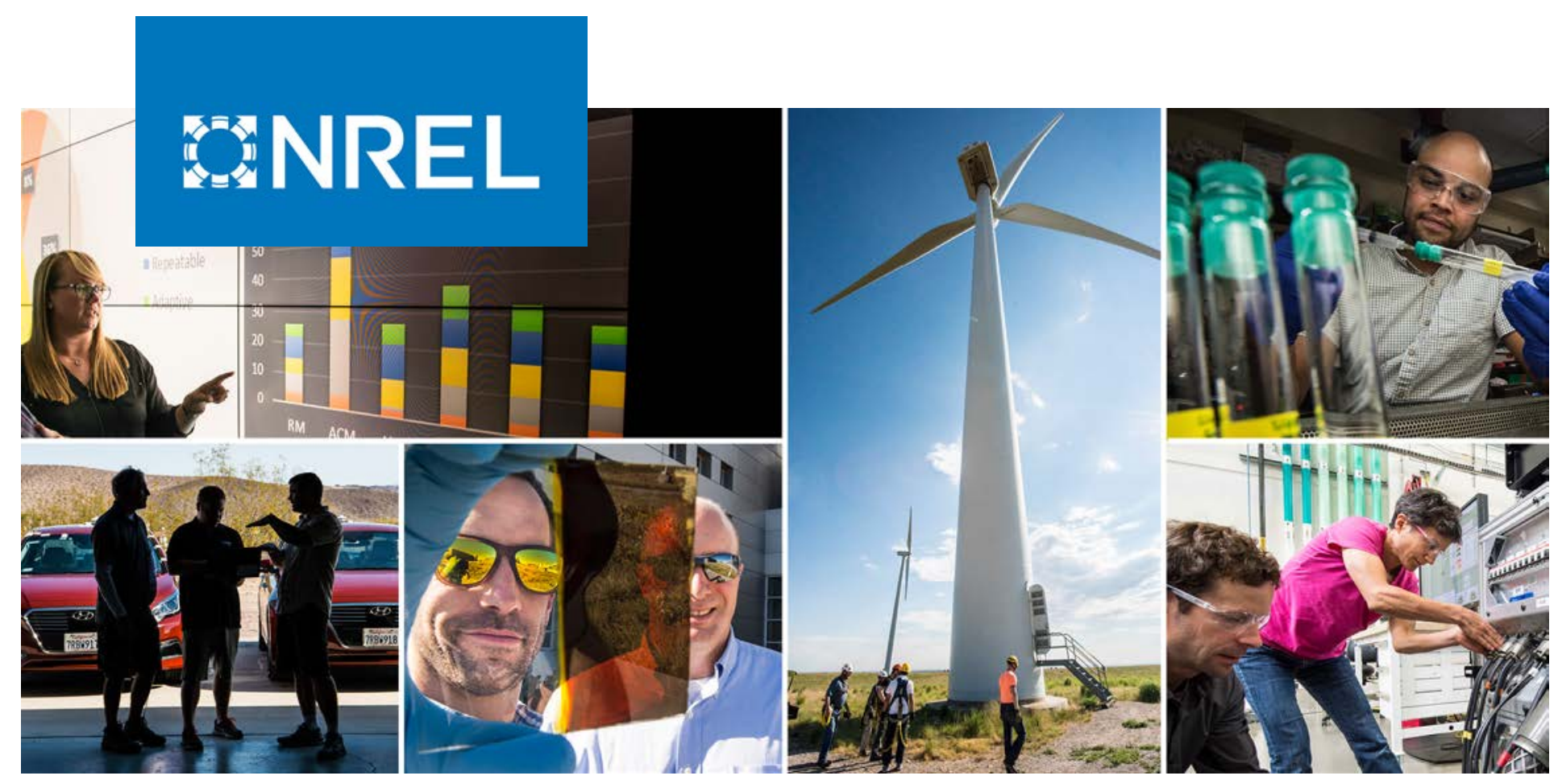

\title{
Equivalent Circuit Models of Voltage- Controlled Dual Active Bridge Converters
}

\section{Preprint}

Rahul Mallik, ${ }^{1}$ Branko Majmunovic, ${ }^{2}$ Satyaki Mukherjee, ${ }^{2}$ Soham Dutta, ${ }^{1}$ Gab-Su Seo, ${ }^{3}$ Dragan Maksimovic, ${ }^{2}$ and Brian Johnson ${ }^{1}$

1 University of Washington

${ }^{2}$ University of Colorado, Boulder

${ }^{3}$ National Renewable Energy Laboratory

Presented at the 2019 IEEE Workshop on Control and Modeling for Power Electronics (IEEE COMPEL)

Toronto, Canada

June 17-20, 2019

NREL is a national laboratory of the U.S. Department of Energy

Office of Energy Efficiency \& Renewable Energy

Operated by the Alliance for Sustainable Energy, LLC

This report is available at no cost from the National Renewable Energy Laboratory (NREL) at www.nrel.gov/publications.
Conference Paper

NREL/CP-5D00-74061

September 2019 


\title{
GNREL
}

\section{Equivalent Circuit Models of Voltage- Controlled Dual Active Bridge Converters}

\section{Preprint}

\author{
Rahul Mallik, ${ }^{1}$ Branko Majmunovic, ${ }^{2}$ Satyaki Mukherjee, ${ }^{2}$ \\ Soham Dutta, ${ }^{1}$ Gab-Su Seo, ${ }^{3}$ Dragan Maksimovic, ${ }^{2}$ \\ and Brian Johnson ${ }^{1}$ \\ ${ }^{1}$ University of Washington \\ 2 University of Colorado, Boulder \\ ${ }^{3}$ National Renewable Energy Laboratory
}

\section{Suggested Citation}

Mallik, Rahul, Branko Majmunovic, Satyaki Mukherjee, Soham Dutta, Gab-Su Seo, Dragan Maksimovic, and Brian Johnson. 2019. Equivalent Circuit Models of VoltageControlled Dual Active Bridge Converters: Preprint. Golden, CO: National Renewable Energy Laboratory. NREL/CP-5D00-74061. https://www.nrel.gov/docs/fy19osti/74061.pdf.

(C) 2019 IEEE. Personal use of this material is permitted. Permission from IEEE must be obtained for all other uses, in any current or future media, including reprinting/republishing this material for advertising or promotional purposes, creating new collective works, for resale or redistribution to servers or lists, or reuse of any copyrighted component of this work in other works.

NREL is a national laboratory of the U.S. Department of Energy Office of Energy Efficiency \& Renewable Energy Operated by the Alliance for Sustainable Energy, LLC

This report is available at no cost from the National Renewable Energy Laboratory (NREL) at www.nrel.gov/publications.

Contract No. DE-AC36-08GO28308
Conference Paper NREL/CP-5D00-74061

September 2019

National Renewable Energy Laboratory 15013 Denver West Parkway Golden, CO 80401

303-275-3000 • www.nrel.gov 


\section{NOTICE}

This work was authored in part by the National Renewable Energy Laboratory, operated by Alliance for Sustainable Energy, LLC, for the U.S. Department of Energy (DOE) under Contract No. DE-AC36-08GO28308. Funding provided by U.S. Department of Energy Office of Energy Efficiency and Renewable Energy Solar Energy Technologies Office and the Laboratory Directed Research and Development (LDRD) Program. The views expressed herein do not necessarily represent the views of the DOE or the U.S. Government. The U.S. Government retains and the publisher, by accepting the article for publication, acknowledges that the U.S. Government retains a nonexclusive, paid-up, irrevocable, worldwide license to publish or reproduce the published form of this work, or allow others to do so, for U.S. Government purposes.

This report is available at no cost from the National Renewable Energy Laboratory (NREL) at www.nrel.gov/publications.

U.S. Department of Energy (DOE) reports produced after 1991 and a growing number of pre-1991 documents are available free via www.OSTI.gov.

Cover Photos by Dennis Schroeder: (clockwise, left to right) NREL 51934, NREL 45897, NREL 42160, NREL 45891, NREL 48097, NREL 46526.

NREL prints on paper that contains recycled content. 


\title{
Equivalent Circuit Models of Voltage-controlled Dual Active Bridge Converters
}

\author{
R. Mallik*, B. Majmunovic ${ }^{\dagger}$, S. Mukherjee ${ }^{\dagger}$, S. Dutta*, G.-S. Seo ${ }^{\ddagger}$, D. Maksimovic ${ }^{\dagger}$, B. Johnson* \\ ${ }^{*}$ Department of Electrical and Computer Engineering, University of Washington, Seattle, WA 98195, USA \\ Email:rmallik@uw.edu,sdutta@uw.edu,brianbj@uw.edu \\ $\dagger$ Department of Electrical, Computer, and Energy Engineering, University of Colorado, Boulder, CO 80309, USA \\ Email: branko.majmunovic@ colorado.edu, satyaki.mukherjee@colorado.edu, maksimov@colorado.edu \\ $\ddagger$ Power Systems Engineering Center, National Renewable Energy Laboratory, Golden, CO 80401, USA \\ Email: gabsu.seo@nrel.gov
}

\begin{abstract}
In this paper, we introduce a new method of modeling voltage-controlled dual active bridge converters as equivalent circuits. What makes the proposed model unique is that the entire closed loop system (converter and control loop) are represented together in one equivalent circuit. Since the full system can be recast as a circuit, this allows for deeper insights on how the closed-loop system performs and for the direct application of circuit analysis techniques. In particular, we reveal how classical control notions can be understood as circuit laws.
\end{abstract}

Index Terms-circuit analysis, control systems, dual-activebridge converters

\section{INTRODUCTION}

Dual active bridge (DAB) converters are widely-used in applications where isolation and high voltage conversion ratios are required [1]. DAB use-cases run the gamut from battery chargers for hybrid electric vehicles [2], to photovoltaic systems [3], and medium voltage grid-connected converters [4]. It is well-known that switch-cycle-averaged converter models are essential for analysis, control design, and reduced computational complexity. Along these lines, we propose a new modeling approach where both the averaged DAB converter and its closed-loop voltage controller are represented together as a unified c ircuit e quivalent. A fter w e r ecast the averaged system as a circuit, we show that its closed-loop characteristics naturally emerge from Kirchhoff's laws. Not only does this circuit-based framework reveal a link between circuit and control laws, but it also gives deeper intuition on how the closed-loop DAB system operates.

Impedance-based methods have emerged as a popular approach to analyze the small-signal dynamics of converter systems [5], [6] as well as for ac distributed power systems [7]. These methods have also been extended to study local instabilities based on input admittance calculation of converters [8].

Funding was provided in part by the DOE Office of E nergy Efficiency and Renewable Energy Solar Energy Technologies Office g rant number DEEE0008346 and by the National Renewable Energy Laboratory, operated by Alliance for Sustainable Energy, LLC, for the U.S. Department of Energy (DOE) under Contract No. DE-AC36-08GO28308. The views expressed in the article do not necessarily represent the views of the DOE or the U.S. Government.
With such a framework, stability can be analyzed in terms of the effective output impedance of a given converter [9] where the output port is modeled as either a Norton or Thevenin equivalent. Although this provides a valuable linkage between the circuit concept of impedance with stability, the converter and its various control loops are not explicitly represented as circuits. In other words, impedance-based approaches do not explicitly reveal the circuit equivalent that captures the feedback and feedforward action. On another related note, virtual oscillator controllers are one special case of when closed-loop converter systems can be represented as a circuit. However, those modeling approaches are not generalized and are applied on a case-by-case basis for oscillator-based controllers.

Generally speaking, classical frequency domain and statespace models provide little physical intuition beyond what can be gleaned from Bode and root-locus plots. To bypass these limitations, we establish a new method for modeling voltagecontrolled converters as circuits. By casting the closed-loop converter system as a circuit, we give physical intuition on how the compensator interacts with the output load, feedback and feedforward sensing paths, and reference signal. After defining the equivalent circuit, we apply Kirchhoff's laws to distill it into a canonical circuit which captures the key inputoutput relations. Finally, we show how superposition along with the voltage and current divider equations directly give us the closed-loop system model without any tedious algebra. Curiously, it emerges that the voltage and current divider equations have a direct mapping to the well-known sensitivity and complementary sensitivity functions that are classically used to analyze closed-loop systems in both the controls [10] and power electronics [11] contexts.

The remainder of the paper is structured as follows: In Section II, we define notation along with converter modeling basics. We derive the equivalent circuit model in Section II. Section IV establishes a one-to-one correspondence between the circuit model with classical control frameworks. Finally, concluding statements are in $\mathrm{V}$.

\section{Modeling Preliminaries}

Consider the DAB circuit in Fig. 1(a) with dc input voltage, $v_{\mathrm{i}}$, a $1: n$ transformer which links the two bridges, transformer 


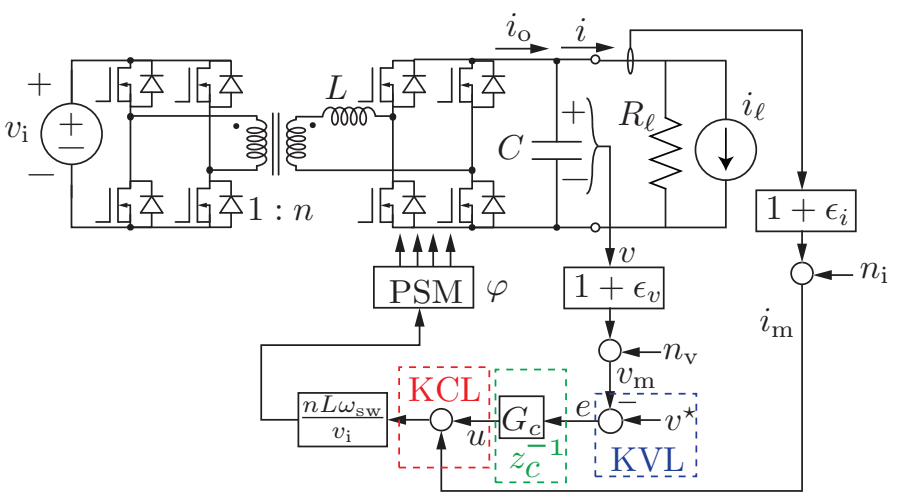

(a)

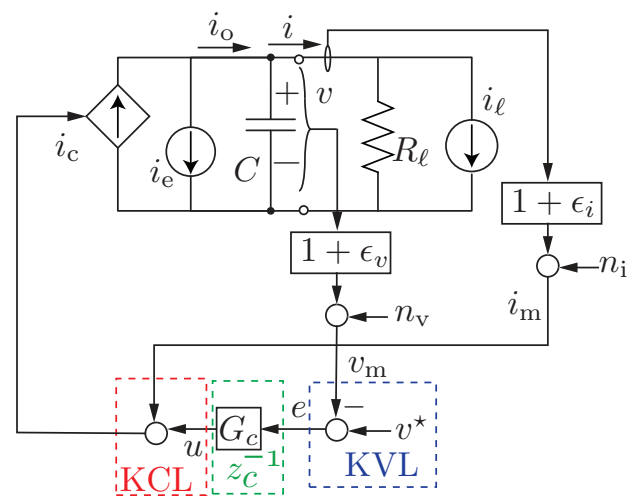

(b)

Fig. 1. We analyze a DAB with voltage control connected to a load. The load is modeled as a nominal resistance in parallel with a current sink. The switched model is in (a) and its switch-cycle-averaged counterpart is in (b).

leakage inductance $L$, and output voltage $v$. The secondary active bridge delivers dc current $i_{\mathrm{o}}$ into the dc output. The terminals of the output capacitance, $C$, define the converter load terminals. We model the external load as a nominal resistance $R_{\ell}$, and a disturbance current source $i_{\ell}$, which captures all other load uncertainties.

A voltage control feedback loop begins with the sensed load voltage and current. We model the non-ideal voltage and current sensor as having an off-nominal scaling factor $\epsilon_{\mathrm{v}}$ and $\epsilon_{\mathrm{i}}$, respectively, and an additive noise $n_{\mathrm{v}}$ and $n_{\mathrm{i}}$, respectively. Hence, the measured load voltage and current, $v_{\mathrm{m}}$ and $i_{\mathrm{m}}$, can be expressed as

$$
\begin{aligned}
& v_{\mathrm{m}}=\left(1+\epsilon_{\mathrm{v}}\right) v+n_{\mathrm{v}} \\
& i_{\mathrm{m}}=\left(1+\epsilon_{\mathrm{i}}\right) i+n_{\mathrm{i}}
\end{aligned}
$$

Reflecting on (1)-(2), ideal sensing is recovered in the limit $\epsilon_{\mathrm{v}}, \epsilon_{\mathrm{i}}, n_{\mathrm{v}}, n_{\mathrm{i}} \rightarrow 0$.

The voltage controller acts on the reference $v^{\star}$ and the compensator, denoted as $G_{\mathrm{c}}$, produces the control effort $u$. As shown in Fig. 1, the load current, $i$, is added to the control effort as a feedforward signal. The DAB utilizes phaseshift modulation (PSM) where the secondary-side phase shift, denoted as $\varphi$, is defined relative to the primary-side switch signal. The switch-cycle-averaged DAB output current is

$$
i_{\mathrm{o}}=\frac{v_{\mathrm{i}} \varphi}{n L \omega_{\mathrm{sw}}}\left(1-\frac{\varphi}{\pi}\right)=i_{\mathrm{c}}-i_{\mathrm{e}}
$$

where

$$
i_{\mathrm{c}}:=\frac{v_{\mathrm{i}}}{n L \omega_{\mathrm{sw}}} \varphi, i_{\mathrm{e}}:=\frac{v_{\mathrm{i}}}{n L \omega_{\mathrm{sw}}} \frac{\varphi^{2}}{\pi}
$$

We consider the setting where the the phase shift, $\varphi$, is kept small such that $\varphi \gg \varphi^{2} / \pi$, and hence $i_{\mathrm{c}} \gg i_{\mathrm{e}}$. To simplify implementation and design, we designate the first-order term, $i_{\mathrm{c}}$, as being directly manipulated by the controller. We apply (4) and scale the control output, $i_{\mathrm{c}}$, by $n L \omega_{\mathrm{sw}} / v_{\mathrm{i}}$ to obtain $\varphi$ (see Fig. 1(a)). Given that the control output is a current signal, we can abstract away the switch modulation and redraw the switched converter in Fig. 1(a) as the circuit in Fig. 1(b) where all variables are averaged over a switch cycle. Averaged quantities are implied throughout from here forward. Note that we retain the second order term, $i_{\mathrm{e}}$, in our averaged model to capture the effect of the small-angle approximation error.

\section{Development of the Equivalent Circuit Model}

Referring to the averaged model in Fig. 1(b), it is evident that the compensator input-side has a voltage difference whereas the output is a current signal. Accordingly, the compensator can be recast as an admittance that translates the voltage difference $v^{\star}-v_{\mathrm{m}}$ into a current, $u$, and the relations on either side of the compensator can be understood via Kirchhoff's laws (see KVL and KCL relations in Fig. 1). This observation allows us to define $z_{\mathrm{c}}^{-1}(s):=G_{\mathrm{c}}(s)$, where $z_{\mathrm{c}}(s)$ is an impedance and its inverse is functionally equivalent to $G_{\mathrm{c}}(s)$. Combining these insights, we redraw the averaged system as the circuit in Fig. 2.

Note that the circuit model in Fig. 2 is an exact representation of the averaged model when both are initialized identically. Reflecting on the circuit model, the reference signal $v^{\star}$, noise $n_{\mathrm{v}}$, and sensor scaling error component $\epsilon_{\mathrm{v}} v$, act as a voltage sources and the error voltage, $e$, is across both $z_{\mathrm{c}}(s)$ and the voltage sensor non-idealities. To summarize, signals on the input-side of the compensator take the form of voltage sources.

Switching focus to the compensator output side, the feedforward current is added to the control effort, $u$. Since the feedforward and control effort are both current signals, we map the control signals in Fig. 1(b) to a corresponding KCL relationship in Fig. 2. Accordingly, the current sensor noise takes the form of a shunt current source and the scaling error effects the controllable current source which models the load current feedforward. Finally, the modeling error, $i_{\mathrm{e}}$, acts as a current source.

\section{A. The Canonical Circuit Equivalent Model}

Once we arrive at the equivalent circuit in Fig. 2, we seek a canonical form that clearly emphasizes key input-output 


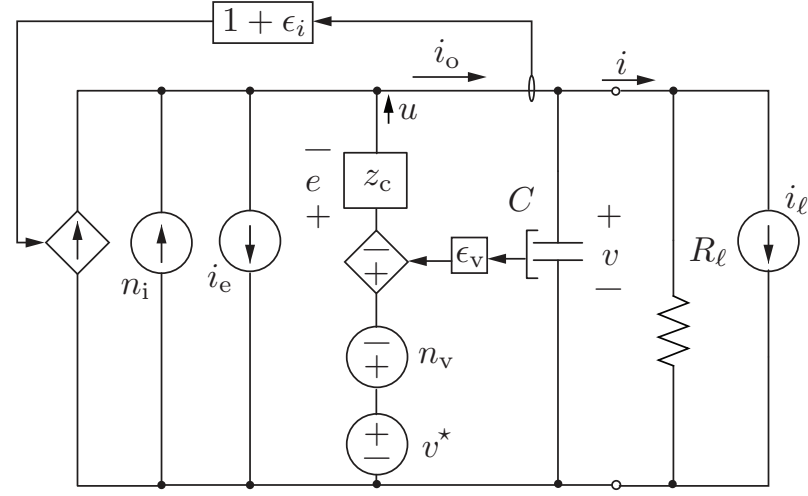

Fig. 2. The equivalent circuit model where the feedback and feedforward control action are translated into equivalent circuit elements. For instance, the setpoint, sensor non-idealities, and modeling errors are interpreted as corresponding voltage and current sources.

relationships. Towards that end, we lump the model nonidealities into composite current and voltage disturbance which we denote as $i_{\mathrm{d}}$ and $v_{\mathrm{d}}$, respectively. In particular,

$$
\begin{array}{r}
i_{\mathrm{d}}=\epsilon_{\mathrm{i}} i_{\ell}+n_{\mathrm{i}}-i_{\mathrm{e}} \\
v_{\mathrm{d}}=\epsilon_{\mathrm{v}} v+n_{\mathrm{v}}
\end{array}
$$

where $i_{\mathrm{d}}$ encapsulates the current sensor scaling error and noise, as well as the small-angle approximation residual. Similarly, $v_{\mathrm{d}}$ contains the voltage sensor non-idealities and noise. Due to scaling error in the feedforward current sensor, an exact cancellation of $R_{\ell}$ is not possible. After accounting for the off-nominal current sensor scaling, the effective load which remains is $R_{\ell} / \epsilon_{\mathrm{i}}$. The canonical circuit is in Fig. 3 where we denote the impedance across the output capacitor and uncanceled load as

$$
z_{\mathrm{p}}(s)=\frac{1}{s C} \| \frac{R_{\ell}}{\epsilon_{\mathrm{i}}}=\frac{R_{\ell} / \epsilon_{\mathrm{i}}}{s C+R_{\ell} / \epsilon_{\mathrm{i}}}
$$

Now that we have arrived at the simplified circuit in Fig. 3 where the disturbances, references voltage, compensator, and load are segregated, we can apply classical circuit analysis methods to compute the closed loop response. Applying superposition along with the voltage and current divider equations, we obtain

$$
\begin{aligned}
v(s) & =\frac{z_{\mathrm{p}}(s)}{z_{\mathrm{p}}(s)+z_{\mathrm{c}}(s)} v^{\star}(s) \\
& -\frac{z_{\mathrm{p}}(s)}{z_{\mathrm{p}}(s)+z_{\mathrm{c}}(s)} v_{\mathrm{d}}(s)+\frac{z_{\mathrm{c}}(s)}{z_{\mathrm{p}}(s)+z_{\mathrm{c}}(s)} z_{\mathrm{p}}(s) i_{\mathrm{d}}(s)
\end{aligned}
$$

The first and second terms captures how the reference voltage sensor errors are dropped across the series-connected load and compensator impedances. The last term highlights how the current signal nonidealities are divide up between the load and compensator branches.

\section{Translating Circuit LaWs into Control LAWS}

Having computed the closed-loop model in (8) with circuit methods only, we next show how classical control relations can

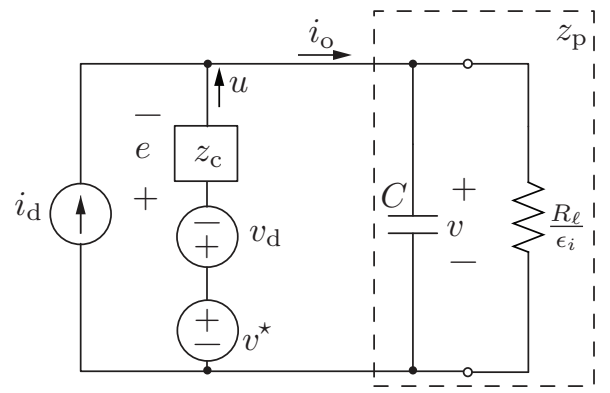

Fig. 3. We obtain the canonical circuit model where all undesirable system characteristics are lumped into one voltage and current source disturbance. This simplified model highlights key input-output relationships and facilitates closed-loop system analysis.

be recovered from our result. This will effectively demonstrate how our circuit-based approach is consistent with classical control frameworks. First we study the closed-loop model and then subsequently show how commonly-used compensator types can be represented as circuits.

\section{A. Closed-loop Models}

To draw a clear linkage with established control methods, we will rewrite our model with notation commonly seen in controls analysis. Recall that the compensator transfer function is related to its equivalent impedance by $G_{\mathrm{c}}(s)=z_{\mathrm{c}}^{-1}(s)$. Next, we designate the effective load impedance as being the plant transfer function $G_{\mathrm{p}}(s):=z_{\mathrm{p}}(s)$, where $z_{\mathrm{p}}(s)$ is given in (7). Once we substitute these definitions into (8) and perform some elementary algebraic manipulations, we recover the wellknown relations [10], [11] below.

$$
\begin{aligned}
& v(s)=\frac{G_{\mathrm{p}}(s) G_{\mathrm{c}}(s)}{1+G_{\mathrm{p}}(s) G_{\mathrm{c}}(s)} v^{\star}(s) \\
& -\frac{G_{\mathrm{p}}(s) G_{\mathrm{c}}(s)}{1+G_{\mathrm{p}}(s) G_{\mathrm{c}}(s)} v_{\mathrm{d}}(s)+\frac{1}{1+G_{\mathrm{p}}(s) G_{\mathrm{c}}(s)} G_{\mathrm{p}}(s) i_{\mathrm{d}}(s)
\end{aligned}
$$

As indicated below, our definitions show that the loop gain is simply the ratio of the plant to controller impedances.

$$
\ell(s):=G_{\mathrm{p}}(s) G_{\mathrm{c}}(s)=\frac{z_{\mathrm{p}}(s)}{z_{\mathrm{c}}(s)}
$$

Adopting controls parlance, we write the closed-loop response in terms of the complementary sensitivity function, $T(s)$, and sensitivity function, $S(s)$, such that

$$
v(s)=T(s) v^{\star}(s)-T(s) v_{\mathrm{d}}(s)+S(s) G_{\mathrm{p}}(s) i_{\mathrm{d}}(s)
$$

where $T(s)=\ell(s) /(1+\ell(s))$ and $S(s)=1 /(1+\ell(s))$. Consider the well-known property $T(s)+S(s)=1$ which implies design tradeoffs between reference tracking and disturbance rejection. If we recall that $T(s)$ and $S(s)$ map to the voltage and current divider equations, it follows that these two circuit quantities, namely $z_{\mathrm{p}}(s) /\left(z_{\mathrm{p}}(s)+z_{\mathrm{c}}(s)\right)$ and $z_{\mathrm{c}}(s) /\left(z_{\mathrm{p}}(s)+z_{\mathrm{c}}(s)\right)$, also sum to unity. This is a fundamental connection between circuit and control analysis that we will revisit later in the paper. 


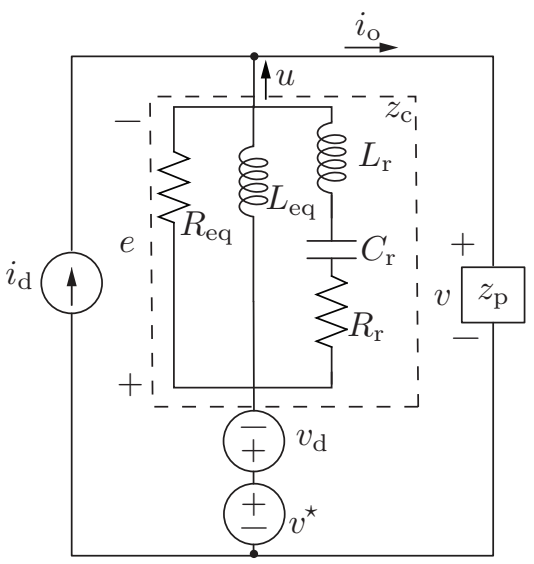

Fig. 4. Equivalent circuit model with a proportional-integral-resonant compensator. The transfer function proportional, integral, and resonant terms translate to resistive, inductive, and $R L C$ tank branches.

\section{B. Circuit Representations of Prototypical Controllers}

Here we consider a simple but generic voltage compensator that can be used in a variety of applications for both constant dc loads and loads which draw pulsating components. In other words, we seek a compensator model that can be applied to a variety of applications. Consider a proportional-integralresonant (PIR) controller [12] where the integral and resonant terms give ideal tracking at $\mathrm{dc}$ and a user-defined resonant frequency $\omega_{\mathrm{r}}$. This gives

$$
G_{\mathrm{c}}(s)=\frac{1}{z_{\mathrm{c}}(s)}=k_{\mathrm{p}}+\frac{k_{\mathrm{i}}}{s}+\frac{k_{\mathrm{r}} s}{s^{2}+2 \xi \omega_{\mathrm{r}} s+\omega_{\mathrm{r}}^{2}}
$$

Next we convert (12) to its corresponding circuit representation. Since $G_{\mathrm{c}}(s)=z_{\mathrm{c}}^{-1}(s)$ and $z_{\mathrm{c}}^{-1}(s)$ is an admittance, we equate (12) to

$$
z_{\mathrm{c}}^{-1}(s)=R_{\mathrm{eq}}^{-1}+\left(s L_{\mathrm{eq}}\right)^{-1}+\left(s L_{\mathrm{r}}+R_{\mathrm{r}}+\frac{1}{s C_{\mathrm{r}}}\right)^{-1}
$$

Furthermore, because $u(s)=z_{\mathrm{c}}^{-1}(s)\left(v^{\star}(s)-v(s)-v_{\mathrm{d}}(s)\right)$ is a current, it follows that each term in (13) corresponds to a parallel circuit branch where the branch currents sum up to $u(s)$. After some manipulations to (13) and a comparison to (12), we obtain

$$
\begin{gathered}
R_{\mathrm{eq}}=\frac{1}{k_{\mathrm{p}}}, \quad L_{\mathrm{eq}}=\frac{1}{k_{\mathrm{i}}} \\
L_{\mathrm{r}}=\frac{1}{k_{\mathrm{r}}}, \quad C_{\mathrm{r}}=\frac{k_{\mathrm{r}}}{\omega_{\mathrm{r}}^{2}}, \quad R_{\mathrm{r}}=\frac{2 \xi \omega_{\mathrm{r}}}{k_{\mathrm{r}}}
\end{gathered}
$$

These relations allow us to straightforwardly translate the parameters of a transfer function $G_{\mathrm{c}}(s)$ to corresponding circuit elements. Ultimately, we arrive at the circuit representation in Fig. 4. Note that PI and PR controllers are easily recovered by eliminating extraneous branches (terms) from the circuit equivalent (transfer function).

\section{Closed-loop Circuit Performance}

Now we consider how classical notions of closed-loop performance translate to the equivalent circuit properties. Traditionally, it is well-understood that ideal reference tracking is obtained for frequencies where $\|\ell(j \omega)\|=$ $\left\|G_{\mathrm{p}}(j \omega) G_{\mathrm{c}}(j \omega)\right\| \rightarrow \infty$, the compensator $G_{\mathrm{c}}(j \omega)$ has high gain, and hence $\|T(j \omega)\| \approx 1$. From a circuits perspective, recall that $\|\ell(j \omega)\|=\left\|z_{\mathrm{p}}(j \omega) / z_{\mathrm{c}}(j \omega)\right\|$ and the voltage divider is equal to $\|T(j \omega)\|=\left\|z_{\mathrm{p}}(j \omega) /\left(z_{\mathrm{p}}(j \omega)+z_{\mathrm{c}}(j \omega)\right)\right\|$. Accordingly, high control gain is equivalent to the setting where $\left\|z_{\mathrm{c}}(j \omega)\right\| \rightarrow 0$, the voltage divider gain approaches unity, and the compensator effectively behaves as a short circuit. Along these lines, ideal tracking at dc and at the resonant frequency, $\omega_{\mathrm{r}}$, are obtained with the inductive branch and resonant branches in Fig. 4 which map to integral and resonant terms in $G_{\mathrm{C}}(s)$. Note that the optional damping term in the resonant branch also prevents controller saturation and wind-up (excessive current in the resonant branch).

Shifting our focus to disturbance rejection, recall that the sensitivity function and current divider expression are equivalent such that $\|S(j \omega)\|=\left\|z_{\mathrm{c}}(j \omega) /\left(z_{\mathrm{p}}(j \omega)+z_{\mathrm{c}}(j \omega)\right)\right\|$. Furthermore, since the voltage and current divider equations sum to unity and hence $S(j \omega)=1-T(j \omega)$, it follows that frequencies with ideal tracking (low value of $\left\|z_{\mathrm{c}}(j \omega)\right\|$ ) also give rejection of the disturbances contained in $i_{\mathrm{d}}(j \omega)$. Expanding on the circuit intuition, this is consistent with $z_{\mathrm{c}}(s)$ shunting $i_{\mathrm{d}}(s)$, which contains current sensor nonidealities and modeling error, away from the load. Lastly, since $S(j \omega)=1-T(j \omega)$, it follows that the controller frequency response (and its circuit equivalent) should be tuned to balance reference tracking as well as rejection of voltage sensor noise in $v_{\mathrm{d}}(j \omega)$. Towards that end, the classical strategy of letting $\|\ell(j \omega)\|$ roll off at high frequencies where noise dominates is tantamount to rising compensator impedance at high frequencies (i.e., $\lim _{\omega \rightarrow \infty}\left\|z_{\mathrm{c}}(j \omega)\right\| \rightarrow \infty$ ). This wellknown strategy is reflected in the inductive branches in $z_{\mathrm{c}}(s)$.

\section{CONCLUSION}

In this paper we introduced a framework to model voltage controlled DABs as circuit equivalents. Compared to prior art, the proposed model is unique since both the averaged converter and control loop are represented together as a unified circuit. Since the controller feedback and feedforward action are represented with an equivalent circuit structure, we reveal a deeper physical intuition of how the closed-loop system operates. Furthermore, we show how the equivalent circuit properties directly map to classical concepts in control analysis. Grounds for future work include the following objectives: i) generalization of circuit equivalent models to other topologies, and ii) application of circuit-based insights to control design.

\section{REFERENCES}

[1] S. Inoue and H. Akagi, "A bidirectional isolated DC-DC converter as a core circuit of the next-generation medium-voltage power conversion system," IEEE Transactions on Power Electronics, March 2007. 
[2] L. Xue, Z. Shen, D. Boroyevich, P. Mattavelli, and D. Diaz, "Dual active bridge-based battery charger for plug-in hybrid electric vehicle with charging current containing low frequency ripple," IEEE Transactions on Power Electronics, vol. 30, pp. 7299-7307, Dec. 2015.

[3] Y. Shi, R. Li, Y. Xue, and H. Li, "Optimized operation of currentfed dual active bridge DC-DC converter for PV applications," IEEE Transactions on Industrial Electronics, vol. 62, pp. 6986-6995, Nov. 2015.

[4] X. She, A. Q. Huang, and R. Burgos, "Review of solid-state transformer technologies and their application in power distribution systems," IEEE Journal of Emerging and Selected Topics in Power Electronics, Sep. 2013.

[5] M. Cespedes and J. Sun, "Impedance modeling and analysis of gridconnected voltage-source converters," IEEE Transactions on Power Electronics, vol. 29, pp. 1254-1261, March 2014.

[6] X. Wang, L. Harnefors, and F. Blaabjerg, "Unified impedance model of grid-connected voltage-source converters," IEEE Transactions on Power Electronics, vol. 33, pp. 1775-1787, Feb. 2018.
[7] J. Sun, "Small-signal methods for AC distributed power systems - A review," IEEE Transactions on Power Electronics, vol. 24, pp. 25452554, Nov. 2009.

[8] L. Harnefors, M. Bongiorno, and S. Lundberg, "Input-admittance calculation and shaping for controlled voltage-source converters," IEEE Transactions on Industrial Electronics, vol. 54, pp. 3323-3334, Dec. 2007.

[9] J. Sun, "Impedance-based stability criterion for grid-connected inverters," IEEE Transactions on Power Electronics, vol. 26, pp. 3075-3078, Nov. 2011.

[10] S. Skogestad and I. Postlethwaite, Multivariable Feedback Control. John Wiley and Sons, 2010.

[11] R. Erickson and D. Maksimovic, Fundamentals of Power Electronics. Springer, 2001.

[12] H. Qin and J. W. Kimball, "Closed-loop control of DC-DC dual-activebridge converters driving single-phase inverters," IEEE Transactions on Power Electronics, Feb. 2014. 